\title{
Extensive postpartum urinary retention successfully treated with clean intermittent catheterization
}

\author{
Paweł Milart, Ewa Beata Wozniakowska, Piotr Czuczwar, Wojciech Wrona, \\ Tomasz Paszkowski \\ $3^{\text {rd }}$ Department of Gynecology, Medical University in Lublin, $3^{\text {rd }}$ Chair and Department of Gynecology, \\ Medical University in Lublin, Poland
}

We present a case of extensive urine retention after vaginal delivery. Postpartum urinary retention occurs in 0.7 to $0.9 \%$ of vaginal deliveries. In the literature, mediolateral episiotomy, epidural analgesia, perineal lacerations, macrosomic birth and prolonged $2^{\text {nd }}$ stage of labor are suggested as risk factors for postpartum urine retention.

A nulliparous 31-year-old patient was admitted to the hospital at 40 weeks of gestation because of uterine contractions. Labor was uncomplicated, the first stage lasted for 12 hours and the second - for 10 minutes. Mediolateral episiotomy was performed. Delivery of the placenta was normal. Four hours after delivery the patient urinated. No complications (obstetrical or urological) were observed during hospitalization. The mother and the newborn were discharged from the hospital on the $4^{\text {th }}$ day after delivery.

On the $5^{\text {th }}$ day after delivery she was consulted in the outpatient's clinic because of acute pain in the lower part of the abdomen, chills and fever up to $39^{\circ} \mathrm{C}$. She reported that the pain and fever were preceded by episodes of urinary incontinence. She was unable to precisely recount the number of micturitions occurring the day before. Urinary retention was diagnosed in the outpatient's clinic by ultrasound. During catheterization 4 liters of urine were evacuated. Twelve hours later, on the $6^{\text {th }}$ day after delivery, the patient again retained 4 liters of urine and therefore catheterization was necessary again. Additionally, acute pain in the lower abdomen and chilling recurred.

With the Foley catheter inserted into the bladder the patient was admitted to the hospital. The patient was particularly monitored concerning the fluid balance. She did not accept discharging from the hospital being catheterized. Oral distigmine bromide was introduced ( $5 \mathrm{mg} / 24 \mathrm{~h}$ for 7 days). After 7 days of catheterization the attempt of catheter removal failed - urine retention recurred. Because the patient retained urine ( 4 liters in 12 hours), ADH was measured, but it was within normal range ( $6.5 \mathrm{ng} / \mathrm{L}, \mathrm{N} 2.5-8.0)$. She did not urinate during the next 12 hours. The patient together with her husband were taught how to perform clean intermittent catheterization $(\mathrm{CIC})$. On the $13^{\text {th }}$ day after delivery the patient was discharged from hospital with the recommendation of $\mathrm{CIC}$ every 3 hours with a 6 hour nocturnal interruption for 3 weeks (days 13-33 after delivery).

The patient presented for follow-up on the $33^{\text {rd }}$ day after delivery, and it was recommended to prolong $\mathrm{CIC}$ every three hours for another month. Urine culture was positive (E. coli $10^{7}$ ), she received two doses of fosfomycin ( $3 \mathrm{~g}$ ). On the $66^{\text {th }}$ day after delivery she reported that the amounts of urine observed during catheterization did not exceed $30 \mathrm{~mL}$. Urine culture was negative. The patient was advised to check postvoiding residual urine volume twice a week. The bladder returned to normal status within three months after delivery.

In the presented case, severe urinary retention occurred after delivery, probably due to the fact that episiotomy was performed. CIC together with distigmine bromide were effective in the treatment of this condition. 